\title{
The antibiotic utilization at the university dentistry clinical center of Kosovo
}

\author{
Fehim Haliti ${ }^{1}$, Naim Haliti ${ }^{2}$, Ferit Koçani ${ }^{3}$, Agim Begzati ${ }^{1}$, Fatmir Dragidella ${ }^{4}$, Luljeta Ferizi ${ }^{1}$, \\ Lumnije Krasniqi ${ }^{1}$, Dafina Doberdoli ${ }^{1}$, Shqiprim Bajrami ${ }^{1}$, Shaip Krasniqi ${ }^{5}$ \\ ${ }^{1}$ Pediatric Dentistry, Department of Stomatology, Faculty of Medicine, University of Prishtina “Hasan Prishtina”, Prishtina, Kosovo \\ ${ }^{2}$ Institute of Forensic Pathology, Faculty of Medicine, University of Prishtina "Hasan Prishtina”, Prishtina, Kosovo \\ ${ }^{3}$ Restorative Dentistry and Endodontology, Department of Stomatology, Faculty of Medicine, University of Prishtina "Hasan \\ Prishtina”, Prishtina, Kosovo \\ ${ }^{4}$ Peridontology and Oral Medicine, Department of Stomatology, Faculty of Medicine, University of Prishtina "Hasan Prishtina”, \\ Prishtina, Kosovo \\ ${ }^{5}$ Institute of Pharmacology and Toxicology and Clinical Pharmacology, Faculty of Medicine, University of Prishtina "Hasan \\ Prishtina”, Prishtina, Kosovo \\ Email: shaip.krasniqi@uni-pr.edu
}

Received 11 October 2013; revised 16 November 2013; accepted 1 December 2013

Copyright (C) 2013 Fehim Haliti et al. This is an open access article distributed under the Creative Commons Attribution License, which permits unrestricted use, distribution, and reproduction in any medium, provided the original work is properly cited.

\begin{abstract}
Antibiotic drugs are a very important drug group considering their frequent use, safety profile and their impact on bacterial resistance induction. Aim: To investigate the utilization of antibiotic drugs at the University Dentistry Clinical Center of Kosovo (UDCCK). Methodology: Retrospective and descriptive study. Patient records were used as a data source collected for a period of 3 months. The total number of registered outpatients in the UDCCK was 1117 patients. The data analysis was performed using the WHO methodology and results were expressed in DDD per 1000 inhabitants per day. Results: The total prescription of antibiotics at UDCCK was 7.18 DDD/ 1000 inhabitants/day. Antibiotics were prescribed for 86 or $7.70 \%$ of patients. The most commonly prescribed antibiotic was Amoxicillin with clavulonic acid 3.12 (43.45\%) DDD, followed by Metronidazole 2.31 DDD and Amoxicillin 1.25 DDD. Erythromycin and Cefalexin were prescribed evidently less compared to other antibiotics (0.38 and 0.12 DDD/1000 inhabitant/day, respectively). Conclusions: The quantitative analysis showed no rationality of prescription of antibiotics in UDCCK. Generally, the use of antibiotics in both departments of UDCCK is higher compared to other countries. We recommend the implementation of restrictive prescription standards for antibiotics.
\end{abstract}

Keywords: Antibiotic; Utilization; ATC/DDD

\section{INTRODUCTION}

In pharmaco-therapeutic clinical practice, antimicrobials are considered to be a group of drugs of essential value, due to their common prescription, therapeuticall success, and their impact on the development of bacterial resistance [1,2]. Their magnitude of prescription is significant not only for in-patients but also for outpatients. Scientific evidence reports that antibiotics are the most commonly prescribed medications in the USA, and they contribute approximately $20 \%-50 \%$ of the total medications used [3]. Antibiotics are an essential group in oral health care as well. The use of these medications requires restrictive prescription by respecting all standards for rational use in order to achieve the highest rate of treatment, less side effects and not to induce the bacterial resistance [4]. The clinical evidence reports that antibiotic prescriptions by dental and medical practitioners are associated with considerable percentage of medication errors and abuse [5, 6]. To achieve the standards for rational prescription of antibiotics by dentists requires knowledge of the bacteriological profile of infections in oral-facial region, choosing of an antibiotic with adequate spectrum of activity and selecting the safest antibiotic possible $[7,8]$. To achieve the standards for rational prescription set in a national drug policy of various countries, there are continuing professional education programs on clinical therapy for medical personnel including strategies for systematic surveys on antibiotic prescribing. All these activities provide feedback information and evaluation of drug prescribing for implementing adequate measures aimed at raising standards for rational prescribing prac- 
tices [9,10].

In daily dentist's services, antibiotic prescription is a frequent practice and a conclusion is drawn that a prescription survey will enable us to have at our disposal information for qualitative critical analysis of antibiotic prescribing [11-13]. Therefore, the purpose of this survey was to perform a pharmacotherapeutical analysis of antimicrobial use in University Dentistry Clinical Center of Kosovo. Simultaneously, we analyzed the structure of diagnoses in order to reveal the quality of the antimicrobial prescription.

\section{MATERIAL AND METHOD}

The survey was a pharmaco-epidemiological, retrospective study performed in the two departments of UDCCK. The data were extracted from electronic patient database and individual hard copy records for each individual patient at the Dept. of Oral Surgery and Dept. of Periodontology, respectively, for a period of (March $3^{\text {rd }}, 2012$ and June $30^{\text {th }}$, 2012) three months. During this period of study a total of 1117 patients were officially recorded (806 at Dept. of Oral Surgery and 311 at Dept. of Periodontology). Patients were identified by their patient record number. For each individual patient we collected information about their gender, diagnosis, daily dose of used antimicrobial and the ATC Antimicrobial Classification $[14,15]$. Parametric testing of the data was performed with the statistics module of Excel ${ }^{\circledR}$ Microsoft $^{\circledR}$ Office ${ }^{\circledR}$ program. The quantitative analysis was performed based on DDD/1000 inhabitants/day (daily defined doses/1000 inhabitants per day) and ATC classification according to WHO, methodologies [16].

\section{RESULTS}

In total, antibiotics were prescribed in 86 patients (7.70\%). There were significant differences in antibiotic prescribing between the departments including differences in diagnoses for witch antimicrobials were prescribed and other parameters as presented below (Table 1). There were no significant differences of the mean for the age of patients between the two departments, however there were reported differences for the gender structure with predominantly female patients in both departments. In the group of patients treated with antibiotics there were significant differences between the departments; $18 \%$ of patients in Dept, of Periodontology vs $3.72 \%$ in the Dept. of Oral Surgery. Brand names were mostly prescribed in both departments $(93.33 \%$ in the Oral Surgery compared to $98.21 \%$ in the Periodontology Department). $1.61 \%$ of patients in the Oral Surgery Department and 3.22\% in Periodontology Department were identified with proven hypersensitivity to penicillin. Figures 1 and $\mathbf{2}$ show the ICD10 coded diagnoses for
Table 1. Demographic data and indicators of antibiotic prescription between oral surgery and periodontology departments.

\begin{tabular}{|c|c|c|c|}
\hline \multicolumn{2}{|c|}{ Department of Oral Surgery } & \multicolumn{2}{|c|}{ Department of Periodontology } \\
\hline \multicolumn{2}{|c|}{ Subject no $=806$} & \multicolumn{2}{|c|}{ Subject no $=311$} \\
\hline \multicolumn{4}{|c|}{ Age } \\
\hline Mean & 36.90 & Mean & 37.16 \\
\hline StDev & 17.42 & StDev & 16.54 \\
\hline Median & 33 & Median & 34 \\
\hline Max & 80 & Max & 80 \\
\hline Min & 33 & Min & 34 \\
\hline \multicolumn{4}{|c|}{ Gender } \\
\hline $\mathrm{F}$ & 448 (55.58\%) & $\mathrm{F}$ & 197 (63.34\%) \\
\hline M & 358 (44.42\%) & M & $114(36.66 \%)$ \\
\hline \multicolumn{4}{|c|}{ Therapy with antibiotics } \\
\hline Yes & $30(3.72 \%)$ & Yes & $56(18 \%)$ \\
\hline No & $776(96.28 \%)$ & No & $255(82 \%)$ \\
\hline \multicolumn{4}{|c|}{ Brand name vs Generic name of antibiotics } \\
\hline Brand name & 28 (93.33\%) & Brand name & 55 (98.21\%) \\
\hline Generic name & $2(6.67 \%)$ & Generic name & $1(1.79 \%)$ \\
\hline \multicolumn{4}{|c|}{ Hypersensitivity against penicillin } \\
\hline \multicolumn{2}{|c|}{ 13/806 (1.61\%) } & \multicolumn{2}{|c|}{ 10/311 (3.22\%) } \\
\hline
\end{tabular}

The structure of patients by ICD10 in the Oral Surgery Department $(\mathrm{n}=807)$

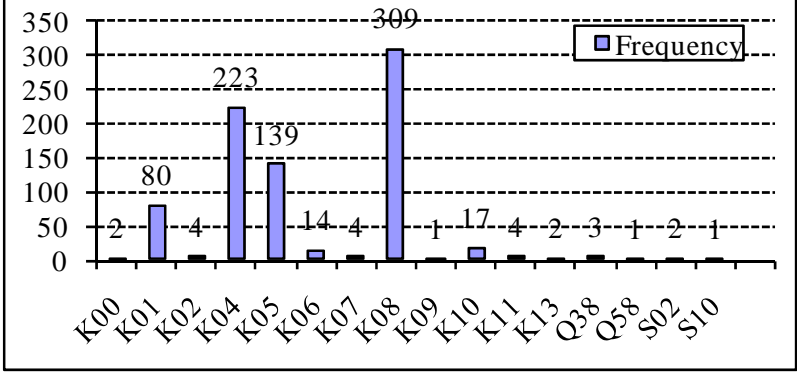

Figure 1. The structure of diagnoses of ICD10 in oral surgery department.

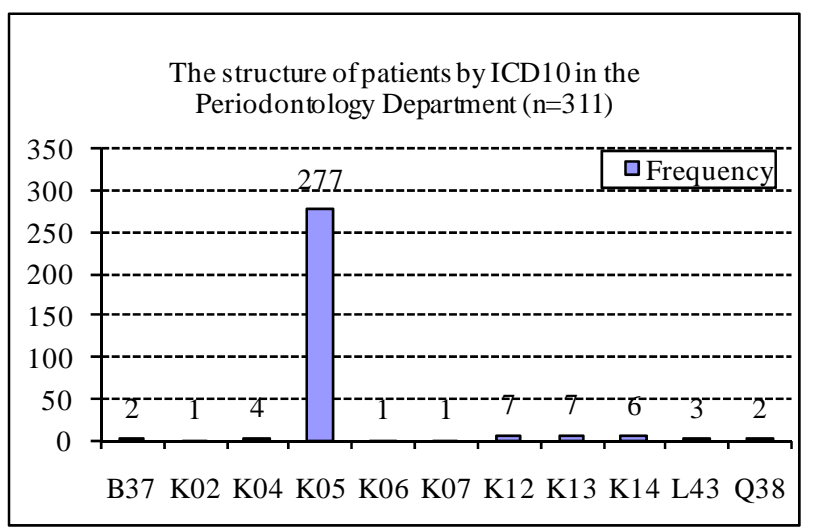

Figure 2. The structure of diagnoses of ICD10 in periodontology department. 
registered patients in both departments. In Oral Surgery department the most frequent diagnoses were K08 (309), K04 (223) and K05 (139) while in the Periodontology Department the majority of diagnoses belonged to K05 category of disease.

The latter diagnosis structure has also influenced the prescription rate of antibiotics and has reflected the differences in total prescription of antibiotics (Table 2). The most prescribed antibiotic was Amoxicillin with Clavulonic acid 3.12 DDD (43.45\%), followed by Metronidazole 2.31 DDD and Amoxicillin 1.25 DDD. Erythromycin and Cefalexin were prescribed evidently less compared to other antibiotics (0.38 or $0.12 \mathrm{DDD} / 1000$ inhabitant/day) (Table 2 and Figure 3). Between the two departments there are significant differences regarding the type and the amount of use of antibiotics.

In the Oral Surgery Department, the utilization of antibiotics is approximately 5 times higher, compared to Periodontology Department (5.97 versus 1.23 DDD/100 inhabitant/day). The prescription rate of Amoxicilin and Clavulonic acid and Metronidazol is significantly higher in Oral Surgery compared to Periodontology Department. However, the difference of Amoxicillin use between the two departments is evidently smaller (remains higher in the Oral Surgery Department).

The use of Erythromycin in Periodontology Department is $0.38 \mathrm{DDD} / 100$ inhabitants/day whereas in Oral Surgery is not prescribed at all. In the Department of Oral Surgery we have noted low rate of prescription of Cefalexin while this antibiotic is not prescribed in the Periodontology Department (Figure 4).

\section{DISCUSION}

The structure of antibiotic use in UDCCK shows a higher level of antibiotic prescription in Oral Surgery Department in particular. A total of four antibiotics were mainly prescribed in both departments of UDCCK. The Dept. of Oral Surgery in line with Amoxicillin and Clavulonic acid and Metronidazole also prescribed Cefalexin. Dept. of Periodontology on the other hand did not prescribe the latter at all, however, they prescribed Erythromycin. Roda R, et al, presented that in Service of Dentistry, Valencia University General Hospital, 8 antibiotics were described in general [17].

Although in the Oral Surgery Department, cases of hypersensitivity to penicillin were identified, and there are no recorded cases of antibiotic usage from macrolide group, which are considered the first choice of treatment for patients hypersensitive to penicillin. This aspect of antibiotherapy remains to be verified; perhaps the statement of hypersensitive patient needs to be further verified with adequate diagnostic methods.

Based on the results of antibiotic use, we can conclude that we found a higher difference between departments and we assume that this is a result of differences in the structure of clinical diagnosis of patients treated in the

Table 2. The antibiotic utilization (DDD/1000 inhabitant/day) at university dentistry clinical center of Kosovo.

\begin{tabular}{clc}
\hline ATC Code & Antibiotic & $\begin{array}{c}\text { DDD/1000 } \\
\text { inhabitant/day }\end{array}$ \\
\hline J01CR02 & Amoxicillin + ac. Clavulonic & 3.12 \\
J01CA04 & Amoxicillin & 1.25 \\
J01XD01 & Metronidazol & 2.31 \\
J01DB01 & Cefalexin & 0.12 \\
J01FA01 & Erythromycin & 0.38 \\
& Total & 7.18 \\
\hline
\end{tabular}

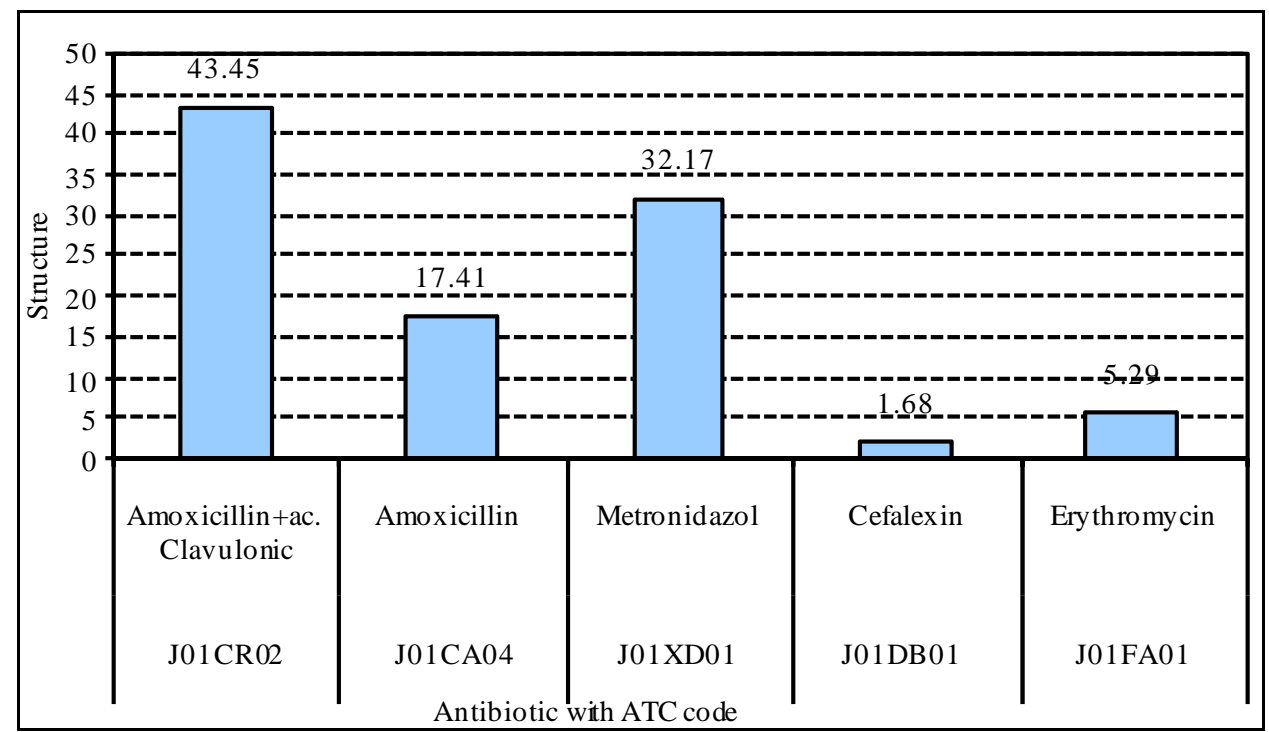

Figure 3. Antibiotic utilization at university dentistry clinical center of Kosovo. 


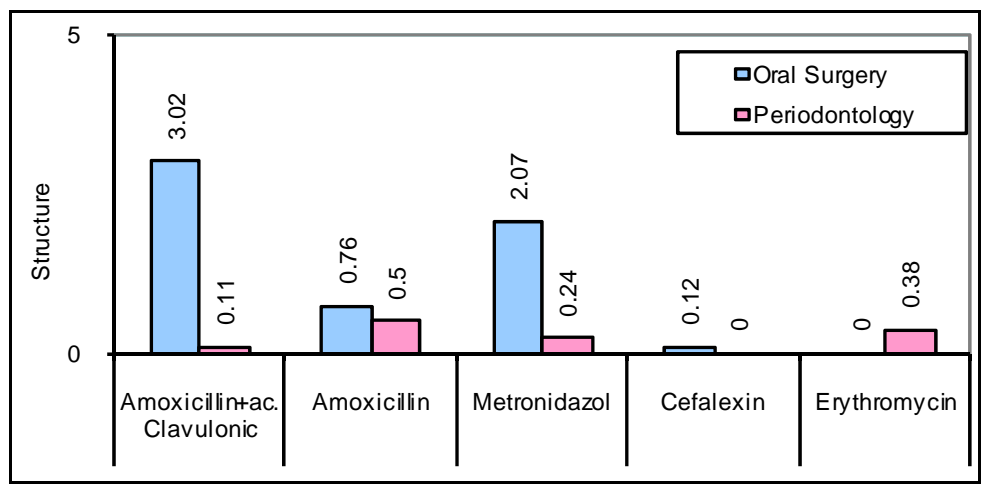

Figure 4. Antibiotic utilization DDD/1000 inhibitants/day by departments of university dentistry clinical center of Kosovo.

two departments.

In both departments of UDCCK, there are still no approved official antimicrobial protocols in the treatment of oral-dental infections. Therefore, we couldn't conduct a qualitative analysis of antibiotic prescribing practices, or the differences in treatment protocols. Furthermore, in the patient records, there are no request notes for microbiology cultures, which is essential in the process of antibiotic selection using sensitivity testing.

The absence of treatment and guidelines for antibiotic prescription also reflects another negative effect, i.e. prescription of the antibiotics by brand name. According to the presented results in both departments, the prescription of antibiotics by their brand names is noted in over $90 \%$ of cases.

The prescription of only four antibiotics in each department shows an irrationality of antibiotics selection. Indeed, the present number of antibiotics in pharmaceutical market gives us a higher opportunity for selection of antibiotics and enabling a higher efficiency and a therapeutic safety.

The antibiotics expenditure in UDCCK is obviously higher, compared to similar medical centers' in other countries. In a survey conducted in different dental centers' of Norway, the rate of DDD/1000 inhabitants units per day in 2004 was 0.5189 DDD, while in 2005 it was 0.60 DDD, which is obviously lower than the antibiotic use in UDCCK. Also in this survey it was reported that 11 different antibiotics were used, phenoxymethylpenicillin being most commonly used narrow spectrum penicillin, then metronidazol, followed by erythromycin, whilst in our survey the most prescribed antibiotics were the broad spectrum penicillin's (Amoxicillin and Clavulonic acid, and amoxicillin) and metronidazol. Although a higher rate of prescription of broad spectrum penicillin compared with the narrow spectrum penicillin is observed also in other countries of Europe like England where the prescription of Amoxicillin was 55.8\% of prescriptions, whereas phenoxymethilpencillin was only $8.2 \%$ [18]. The higher prescription of metronidazole is consistent with the prescription rate of dental practice in other countries, considering that metronidazole is an efficient antibiotic for the treatment of anaerobic oral-dental infections.

In all dental centers of Norway involved in the survey, the metronidazole was prescribed between $6.3 \%$ to $6.9 \%$ of all prescriptions, however, in Great Britain the prescription of metronidazole is significantly higher.

While considering the above presented facts, we conclude that the expenditure of antibiotics generally in both departments of UDCCK is higher especially in Oral Surgery Department [19].

The prescription of antibiotics is not preceded by microbiological testing of samples and in the UDCCK the antibiotic protocols are not in use.

Generally, we recommend performing surveys for determination of microbial sensitivity and bacterial resistance of organisms causing oral-facial infections. Moreover, we recommend the approval of treatment protocols for oral-dental infections, and organization of trainings for antibiotic use in dental practice in the framework of continuing medical education.

\section{REFERENCES}

[1] Col, N.F. and O’Conor, R.W. (1987) Estimating worldwide current antimicrobial usage: Report of Task Force 1. Reviews of Infectious Diseases, 9, S232-S243. http://dx.doi.org/10.1093/clinids/9.Supplement_3.S232

[2] Weber, J.T. and Courvalin, P. (2005) An emptying quiver: Antimicrobial drugs and resistance. Emerging Infectious Diseases, 11, 791-793. http://dx.doi.org/10.3201/eid1106.050471

[3] Pestotnik, S.L., Classer, D.C., Evans, R.S. and Burke, J.P. (1996) Implementing antibiotic practice guidelines through computer-assisted decision support: Clinical and financial outcomes. Annals of Internal Medicine, 124, 884-890. http://dx.doi.org/10.7326/0003-4819-124-10-199605150$\underline{00004}$

[4] Hay, A.D., Thomas, M. and Montgomery, A. (2005) The relationship between primary care antibiotic prescribing 
and bacterial resistance in adults in the community: A controlled observational study using individual patient data. Journal of Antimicrobial Chemotherapy, 56, 146153. http://dx.doi.org/10.1093/jac/dki181

[5] Prober, C.G. and Gold, R. (1980) Antibiotic abuse: Spare the child. Canadian Medical Association Journal, 122, 79.

[6] Kunin, C.M., Johandsen, K.S. and Warning, A.M. (1990) Report of a symposium on use and abuse of antibiotics worldwide. Reviews of Infectious Diseases, 12, 12-19. http://dx.doi.org/10.1093/clinids/12.1.12

[7] Goossens, H., Guillemot, D., Ferech, M., Schlemmer, B., Costers, M., van Breda, M., Baker, L.J., Cars, O. and Davey, P.G. (2006) National campaigns to improve antibiotic use. European Journal of Clinical Pharmacology, 62, 373-379. http://dx.doi.org/10.1007/s00228-005-0094-7

[8] American Dental Association and American Academy of Orthopedic Surgeons (2003) Antibiotic prophylaxis for dental patients with total joint replacements. Journal of American Dental Association, 134, 895-899.

[9] McGowan, B., Bergin, C., Bennett, K. and Barry, M. (2008) Utilization of antibiotic therapy in community practice. Irish Medical Journal, 101, 273-276.

[10] Goossens, H., Guillemot, D., Ferech, M., Schlemmer, B., Costers, M., van Breda, M., Baker, L.J., Cars, O. and Davey, P.G. (2006) National campaigns to improve antibiotic use. European Journal of Clinical Pharmacology, 62, 373-379. http://dx.doi.org/10.1007/s00228-005-0094-7

[11] Pallasch, T.J. and Slots, J. (2000) Antibiotic prophylaxis and the medically compromised patient. Periodontology, 10, 107-138. http://dx.doi.org/10.1111/j.1600-0757.1996.tb00071.x

[12] Van de Sande-Bruinsma, N., Grundmann, H., Verloo, D., Tiemersma, E., Monen, J., Goossens, H. and Ferech, M. (2008) Antimicrobial drug use and resistance in Europe. Emerging Infectious Diseases, 14, 1722-1730. http://dx.doi.org/10.3201/eid1411.070467

[13] Al-Haroni, M. and Skaug, N. (2006) Knowledge of prescribing antimicrobials among Yemeni general dentists. Acta Odontologica Scandinavica, 64, 274-280. http://dx.doi.org/10.1080/00016350600672829

[14] WHO Collaborating Centre to Drug Statistics Methodology (2007) The ATC/DDD system. http:www.whocc.no/atcddd/

[15] (2002) WHO Collaborating Centre for drug statistics methodology. ATC Index with DDDs, Oslo.

[16] (2002) WHO Collaborating Centre for drug statistics methodology. Guidelines for ATC Classification and DDD Assignment, Oslo.

[17] Poveda Roda, R., Bagan, J.V., Sanchis Bielsa, J.M. and Carbonell Pastor, E. (2007) Antibiotic use in dental practice. A review. Medicina Oral Patologia Oral y Cirugia Bucal, 12, E186-E192.

[18] Al-Haroni, M. and Skaug, N. (2007) Incidence of antibiotic prescribing in dental practice in Norway and its contribution to national consumption. Journal of Antimicrobial Chemotherapy, 59, 1161-1166. http://dx.doi.org/10.1093/jac/dkm090

[19] Palmer, N.O., Martin, M.V., Pealing, R., et al. (2000) An analysis of antibiotic prescriptions from general dental practitioners in England. Journal of Antimicrobial Chemotherapy, 46, 1033-1035. http://dx.doi.org/10.1093/jac/46.6.1033 